\title{
Review Article \\ Obstructive Sleep Apnea, Oxidative Stress, and Cardiovascular Disease: Evidence from Human Studies
}

\author{
Hans-Joachim Eisele, Philipp Markart, and Richard Schulz \\ Department of Pneumology, University Hospital of Marburg, Campus Fulda, Pacelliallee 4, 36043 Fulda, Germany \\ Correspondence should be addressed to Richard Schulz; richard.schulz@klinikum-fulda.de
}

Received 17 December 2014; Revised 6 May 2015; Accepted 28 May 2015

Academic Editor: Jacek Zielonka

Copyright (C) 2015 Hans-Joachim Eisele et al. This is an open access article distributed under the Creative Commons Attribution License, which permits unrestricted use, distribution, and reproduction in any medium, provided the original work is properly cited.

\begin{abstract}
Obstructive sleep apnea (OSA) is a frequent disease mainly affecting obese people and caused by repetitive collapse of the upper airways during sleep. The increased morbidity and mortality of OSA are mainly thought to be the consequence of its adverse effects on cardiovascular (CV) health. In this context, oxidative stress induced by nocturnal intermittent hypoxia has been identified to play a major role. This is suggested by biomarker studies in OSA patients showing excessively generated reactive oxygen species from leukocytes, reduced plasma levels of nitrite and nitrate, increased lipid peroxidation, and reduced antioxidant capacity. Biopsy studies complement these findings by demonstrating reduced endothelial nitric oxide synthase expression and increased nitrotyrosine immunofluorescence in the vasculature of these patients. Furthermore, oxidative stress in OSA correlates with surrogate markers of CV disease such as endothelial function, intima-media thickness, and high blood pressure. Continuous positive airway pressure therapy reverses oxidative stress in OSA. The same may be true for antioxidants; however, more studies are needed to clarify this issue.
\end{abstract}

\section{Introduction}

Obstructive sleep apnea (OSA) is the most common form of sleep-disordered breathing (SDB) and represents a major public health problem. It is caused by repetitive collapse of a narrow upper airway during sleep [1]. The main risk factors of the disease are obesity, male gender, and advanced age [2] but it may also occur in lean subjects, women, and children. Earlier studies have reported that OSA affects 2\% and $4 \%$ of middle-aged women and men, respectively [3]. However, owing to the global obesity epidemic, these features might even underestimate the actual prevalence of OSA as suggested by more recent data from epidemiological studies [4].

The episodes of OSA are terminated by arousals. These events disturb normal sleep architecture and can lead to excessive daytime sleepiness in many patients. Furthermore, the risk of traffic accidents is increased. Finally, OSA is linked to enhanced cardiovascular (CV) morbidity and mortality [5]. The gold standard in the diagnosis of OSA is polysomnography and the mainstay of its treatment is continuous positive airway pressure (CPAP) therapy. By creating a pneumatic splint, CPAP rapidly eliminates upper airway collapse and thereby also most sequelae of the disease [6].

The aim of the current review article is to summarize the evidence for oxidative stress being present in humans with OSA and its relation to CV disease. Of note, this has been paralleled by the results of animal studies evaluating the effects of intermittent hypoxia (IH) on the CV system of rodents. For further details, the interested reader is referred to an earlier article we have published in this journal [7].

\section{Clinical Spectrum of OSA-Associated Cardiovascular Diseases}

During the night, blood pressure (BP) surges can be observed in both the systemic and pulmonary circulation [8]. Similarly, cyclical variations of the heart rate emerge (i.e., sinus tachycardia/bradycardia [9]). Furthermore, cardiac rhythm disturbances may occur [10]. These include sinus arrests, 
atrioventricular conduction blocks, atrial fibrillation, and ventricular arrhythmias. It is hypothesized that through these arrhythmias OSA can cause sudden cardiac death; however, the evidence for this is still somewhat circumstantial [11].

The deleterious effects of OSA on the CV system are carried over into daytime hours. Thus, up to one half of these patients suffer from arterial hypertension. The OSAassociated arterial hypertension is characterized by a nondipping 24-hour BP pattern and a high percentage of refractory and masked hypertension [12-14]. Mainly through its pressor effects, OSA increases the risks for stroke, heart failure, and myocardial infarction [5]. In addition, this may lead to more rapid expansion of aortic aneurysms [15]. OSA can also cause pulmonary hypertension; however, this occurs in a lower proportion of patients and usually is not very pronounced [16]. Furthermore, it may be a risk factor for thromboembolic disorders such as deep vein thrombosis and pulmonary embolism [17]. Finally, otherwise healthy OSA patients can already display more subtle CV changes such as endothelial dysfunction and vascular remodeling $[18,19]$.

CV risk in OSA depends on the severity of SDB; that is, those patients with an apnea-hypopnea-index exceeding 30 per hour of sleep are mainly affected [5]. Most studies evaluating CV morbidity and mortality in OSA have primarily enrolled typical sleep laboratory cohorts, that is, middle-aged obese men.

However, more recent data show that $\mathrm{CV}$ risk is also increased in other subsets of OSA patients such as women and the elderly $[20,21]$. Of note, this is independent from established CV risk factors such as advanced age, obesity, smoking, and metabolic disorders.

It is well known that sleep characteristics such as sleep duration and the ability to fall asleep or maintain normal sleep can impact on CV health $[22,23]$. Consequently, those OSA patients who suffer from insomnia or excessive daytime sleepiness have a higher CV risk than those who do not [24].

\section{Pathophysiology of OSA-Associated Cardiovascular Diseases}

Over the last two decades, the intermediary pathways linking OSA to CV disease have been further elucidated. The main trigger of these metabolic and cellular changes is $\mathrm{IH}$, that is, the nocturnal hypoxia-reoxygenation events accompanying OSA. Other OSA-associated stimuli such as hypercapnia, arousals, and intrathoracic pressure swings may also play significant roles; however, this has been less extensively studied.

One major pathogenic mechanism of $\mathrm{CV}$ disease in OSA is sympathetic activation [25]. It is mediated by enhanced chemoreflex activity in the carotid body. Furthermore, inflammatory changes within the vasculature and the adipose tissue which are due to $\mathrm{NF} \kappa \mathrm{B}$ activation have been described $[26,27]$. Additional pathways include the upregulation of hypoxia-sensitive endothelial-derived peptides such as endothelin and vascular endothelial growth factor, enhanced coagulation, and decreased fibrinolysis as well as disturbed vascular repair mechanisms [28-31]. Finally,

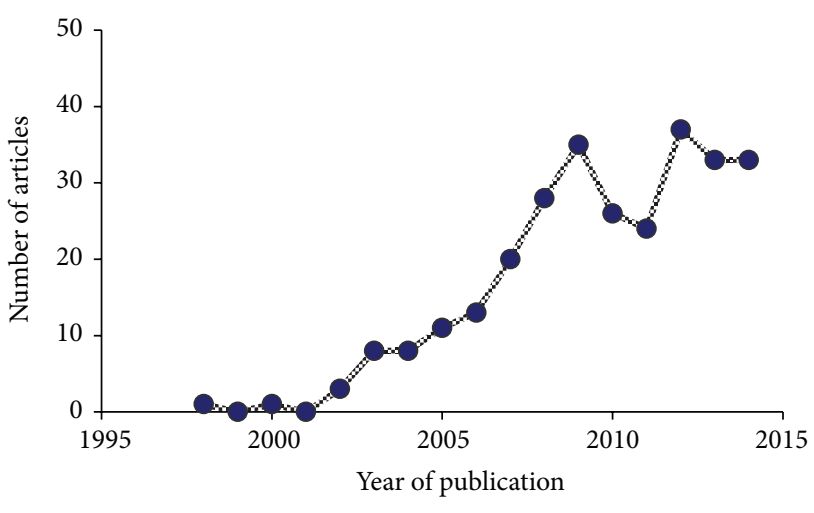

FIgUre 1: Publications found in Pubmed with the search terms of "sleep apnea," "cardiovascular" and "oxidative stress."

OSA is linked to insulin resistance and dyslipidemia which may also contribute to the emergence of CV disease [32, 33].

Another potential mechanism of $\mathrm{CV}$ disease in OSA is an increased oxidative stress. Under physiologic conditions, there is a balance between aggressive and protective factors influencing redox control. The term "oxidative stress" refers to an imbalance between reactive oxygen species (ROS) and reactive nitrogen species production and the system of antioxidant defense resulting in a serious disorder of redox homeostasis [34]. Oxidative stress has been reported to underlie the process of aging and plays a role in the pathogenesis of such different diseases as cancer, chronic inflammatory, and neurodegenerative disorders [35]. Furthermore, there is a strong correlation between oxidative stress and CV diseases such as atherosclerosis, hypertension, and endothelial dysfunction [36].

\section{Evidence for Oxidative Stress in Humans with OSA}

In the following paragraphs, the various aspects of oxidative stress in patients with OSA will be discussed in more detail. For this purpose, a Pubmed search was performed with the search terms "sleep apnea," "cardiovascular" and "oxidative stress." As can be seen from Figure 1 this search yielded a growing number of publications dating back to the late 1990s. Overall, a total of 292 entries were retrieved. Only full-length original publications dealing with human subjects with OSA were taken into consideration. With a few exceptions, replication studies as well as those not evaluating the effects of CPAP therapy on oxidative stress parameters were excluded. Following this strategy, 36 publications were selected for this review article.

4.1. Methodological Aspects of Studies. The studies exploring oxidative stress in OSA have employed different methodological approaches. Most commonly, biomarkers of oxidative stress were determined in peripheral venous blood samples obtained after sleep. Other studies aimed to integrate $\mathrm{CV}$ surrogate markers such as vasoreactivity and 
intima-media thickness (IMT). Finally, some investigators have applied more invasive procedures, that is, vascular biopsies.

Most studies enrolled patients with OSA and controls without SDB and measured the aforementioned parameters before and after CPAP therapy. The majority of these studies had low patient numbers. Furthermore, most of them were not randomized and uncontrolled; that is, they did not have a treatment arm with, for example, sham CPAP (i.e., with subtherapeutical pressure). Finally, many earlier studies recruited patients with comorbidities which might cause oxidative stress by itself. Therefore, it is recommended to restrict study participation to otherwise healthy, nonobese, and nonsmoking OSA patients when performing investigations in this field.

It should be acknowledged that the studies evaluating oxidative stress in OSA have not yielded unequivocal results. This may be due to varying severities of $\mathrm{IH}$ in the patients studied (i.e., frequency and extent of nocturnal oxygen desaturations [37-39]). Patient characteristics as age, gender, and body weight may also have an influence; however, this has not yet been substantiated. Furthermore, as mentioned above discrepancies in comorbidities may introduce some bias into analyses of oxidative stress parameters in these patients. Finally, differences in study design may play important roles in this context (i.e., full-night polysomnography versus splitnight protocols [40]).

4.2. Enhanced Release of Superoxide from Leukocytes. The first studies demonstrating the presence of oxidative stress in humans with OSA were performed in our laboratory at the University of Giessen Lung Center, Germany, and at the University of Haifa, Israel, by the group of Lena and Peretz Lavie [41, 42]. It was observed that circulating neutrophils and monocytes of OSA patients exhibited markedly enhanced in vitro release of superoxide radical anions. This was the case in response to different stimuli such as formylmethionylleucylphenylalanine and phorbol myristate acetate but also in the unstimulated state. The increased oxidative burst of leukocytes in OSA is due to an activation of NADPH oxidase (NOX). The expression of this major superoxidegenerating enzyme is upregulated in leukocytes from OSA patients. This was shown by measuring p22phox mRNA in peripheral blood mononuclear cells [43]. Furthermore, the serum levels of soluble NOX2-derived peptide, a marker of NOX activation by blood cells, decrease after CPAP therapy in patients with OSA [44].

4.3. Reduced Bioavailability of Nitric Oxide. Nitric oxide (NO) is synthesized from the amino acid L-arginine by the action of NO synthases (NOS). They occur in three different isoforms: endothelial, inducible, and neuronal. Endothelial NOS (eNOS) is the enzyme responsible for vascular NO production [45]. Importantly, some naturally occurring compounds can interfere with NO formation. For example, asymmetric dimethylarginine (ADMA) inhibits eNOS and arginase metabolizes L-arginine to L-ornithine and urea [46].
NO is the main endothelial-derived vasodilator. This effect is mediated by its second messenger guanosine $3^{\prime}, 5^{\prime}$ cyclic monophosphate [47]. NO also possesses other vasoprotective properties such as inhibition of platelet aggregation and adhesion molecule expression. The consequences of NO deficiency are, for example, elevated BP and enhanced atherosclerosis [48].

Through various pathways, oxidative stress can reduce NO bioavailability. First, superoxide directly interacts with NO resulting in the formation of peroxynitrite [49]. Second, the degradation of the eNOS cofactor tetrahydrobiopterin is accelerated [50]. Third, the activity of the enzyme dimethylarginine dimethylaminohydrolase is decreased, leading to higher levels of ADMA [51].

Due to its very short half-life of only a few seconds, it is challenging to measure NO itself. More feasible is the determination of its oxidation products nitrite and nitrate. In patients with OSA it was shown that serum levels of nitrite and nitrate are reduced when compared with controls without SDB [52-54]. The decreased nitrite and nitrate pool in OSA is probably due to decreased NO biosynthesis. This is suggested by the findings of increased plasma concentrations of ADMA and arginase in these patients [55-57].

4.4. Increased Oxidation of Lipids, Proteins, and DNA. Excessively generated ROS may lead to increased oxidation of biological compounds such as lipids, proteins, and DNA. Thus, OSA patients show evidence for increased lipid peroxidation as mirrored by elevated plasma concentrations of thiobarbituric acid reactive substances $[58,59]$. In addition to inflammatory changes in response to $\mathrm{IH}$, this may be responsible for accelerated atherosclerosis in affected patients.

Moreover, increased concentrations of 8-isoprostane can be found in exhaled breath condensate of OSA patients [60]. The isoprostanes are prostaglandin-like compounds formed in vivo from the free radical-catalyzed peroxidation of essential fatty acids (primarily arachidonic acid [61]). They enhance vasoconstrictor tone and may thus contribute to the development of arterial hypertension in OSA.

Some authors have reported on higher plasma advanced oxidation protein products in OSA versus non-OSA patients $[62,63]$. DNA oxidation also takes place in OSA as suggested by the finding of increased urinary excretion of 8hydroxy- $2^{\prime}$-deoxyguanosine in patients with severe versus mild-to-moderate OSA [64]. In addition, the levels of DNA damage in peripheral blood lymphocytes as assessed by the cytokinesis-blocked micronucleus assay are increased in OSA [65]. Finally, a significant decrease in mitochondrial DNA copy number was observed in genomic DNA isolated from whole blood of OSA patients [66]. The pathophysiological significance of these changes in the context of OSA-associated $\mathrm{CV}$ disease awaits to be determined.

4.5. Reduced Antioxidant Capacity. The effects of ROS may be counterbalanced by antioxidant substances such as glutathione and vitamins $\mathrm{A}, \mathrm{C}$, and $\mathrm{E}$. Some studies suggest that this defense line against oxidative stress is impaired in untreated patients with OSA. Antioxidant capacity as 
measured by the trolox equivalent antioxidant capacity assay was found to be reduced in OSA [67]. Furthermore, plasma total antioxidant status and levels of vitamins $\mathrm{A}$ and $\mathrm{E}$ were lower in OSA patients versus controls [68]. Finally, the antioxidant properties of serum albumin were shown to be impaired in OSA [69].

Oxidative stress in OSA may also stem from reduced activity of antioxidant enzymes. Thus, lower plasma levels of superoxide dismutase (SOD) have been described in OSA versus non-OSA patients [43, 70]. Similarly, a study evaluating microarray measures of gene transcript levels noted changes in genes encoding for antioxidant enzymes, that is, SOD and catalase [71]. Finally, OSA patients exhibit a decrease in paraoxonase-1, that is, an enzyme which protects lipoproteins from oxidation and thereby exerts antiatherogenic effects [59].

4.6. Vascular Biopsy Studies. Up to now, only a few studies have directly evaluated oxidative stress in the vasculature of OSA patients. This may be due to the reluctance of patients to have biopsies performed and also to the limited availability of these techniques. Jelic et al. harvested endothelial cells by introducing vascular guidewires into forearm veins. When compared to controls without $\mathrm{SDB}$, the cells obtained from OSA patients showed reduced expression of eNOS and increased nitrotyrosine immunofluorescence [72]. Quite similar findings were later reported by Patt et al. who investigated arterioles isolated from forearm subcutaneous biopsies [73]. In a most recent study, endothelial tissue was obtained from gluteal biopsies of OSA and control subjects and eNOS uncoupling was identified as a novel pathway of OSA-associated endothelial dysfunction [74].

4.7. Correlation of Oxidative Stress in OSA with Surrogate Markers of CV Disease. OSA patients may exhibit endothelial dysfunction, that is, a reduction of endothelial-dependent vasorelaxation. This has been shown by various techniques investigating vasoreactivity as, for example, venous occlusion plethysmography and measurements of flow-mediated vasodilation (FMD) of the brachial artery $[18,75]$. Endothelial dysfunction is a precursor lesion for both atherosclerosis and arterial hypertension and can already be detected in OSA patients without clinically overt CV disease.

Since endothelial-dependent vasodilation is mainly the result of NO release, it is not surprising that OSA patients with lower circulating levels of nitrite and nitrate have more severely impaired FMD [76]. Furthermore, the expression of eNOS correlates with FMD in these patients [72]. Finally, inverse relationships between \%FMD and circulating levels of ADMA, soluble NOX2-derived peptide, and isoprostanes have been found $[76,77]$. Taken together, these data clearly show that oxidative stress mechanisms underlie endothelial dysfunction in OSA.

Ultrasonographic studies have shown that OSA patients have greater IMT of their common carotid arteries when compared with non-OSA control subjects and that this is related to the degree of nocturnal hypoxia [19]. IMT reflects the early stages of atherosclerosis and predicts the risks of future stroke and myocardial infarction. Up to now, there is only one study which has investigated the correlation between oxidative stress and IMT in OSA. Monneret et al. found that the IMT of these patients was greater with higher urinary levels of isoprostanes [78].

Likewise, there is a paucity of data on the relationship between oxidative stress and BP in patients with OSA. Ip et al. have reported that the serum levels of nitrite and nitrate are inversely correlated with BP values in OSA [53]. Insofar, the NO deficiency characteristic of OSA may constitute one pathophysiological pathway for the development of arterial hypertension in these patients; however, it is felt that more investigations are required to support this hypothesis.

4.8. Effects of Therapeutical Interventions. As already stated, many of the studies evaluating the effects of CPAP therapy on oxidative stress in OSA had methodological drawbacks such as low patient numbers, inclusion of patients with comorbidities and observational design. Nevertheless, there is no doubt that CPAP therapy ameliorates or even eliminates oxidative stress in OSA. Thus, most changes in biomarker studies were rapidly reversible after only some nights of CPAP (Table 1). For instance, it was reported that CPAP therapy decreases the release of superoxide from leukocytes and increases circulating levels of nitrite and nitrate [41, $42,52-54]$. In addition, the immunohistochemical changes in endothelial cells consistent with oxidative stress were reversible after 1-3 months of CPAP therapy [72, 73]. These findings argue for more structural effects of CPAP therapy on the vasculature which may translate into a long-lasting suppression of oxidative stress in affected individuals.

On the other hand, it must be realized that a significant proportion of OSA patients is not able or willing to use CPAP therapy. Pilot studies suggest that antioxidants may be used to prevent or treat $\mathrm{CV}$ disease in such patients. We have shown that intravenous administration of the antioxidant vitamin $C$ can acutely improve vasoreactivity in OSA [79]. This observation was later confirmed by another group [80]. Furthermore, it was found that the xanthine oxidase inhibitor allopurinol when given orally over two weeks increases FMD in OSA [81].

4.9. Prospects for Future Research. Despite the advancements highlighted in this review, we believe that more research is needed to ascertain a causal role of oxidative stress in OSAassociated CV disease. Appropriate studies should either investigate highly selected OSA patients (i.e., lean subjects without any comorbidities) or large cohorts allowing for statistical control of confounders. Furthermore, randomized controlled designs as, for example, with sham-CPAP treatment should be applied if possible.

One important area of future research certainly is the correlation of oxidative stress biomarkers in OSA with $\mathrm{CV}$ read-out parameters such as FMD, IMT, and BP. Long-term studies could possibly also evaluate the association with more robust $\mathrm{CV}$ endpoints such as the occurrence of myocardial 
TABLE 1: Overview of selected biomarker studies of oxidative stress in OSA.

\begin{tabular}{|c|c|c|c|c|c|}
\hline $\begin{array}{l}\text { Biomarker } \\
\text { investigated }\end{array}$ & $\begin{array}{c}\text { Author/year [reference } \\
\text { number] }\end{array}$ & $\begin{array}{c}\text { OSA } / n= \\
\text { comorbidities yes/no }\end{array}$ & $\begin{array}{c}\text { Controls } / n= \\
\text { comorbidities yes/no }\end{array}$ & $\begin{array}{l}\text { Change of } \\
\text { biomarker }\end{array}$ & $\begin{array}{l}\text { Effects of } \\
\text { CPAP }\end{array}$ \\
\hline PMN oxidative burst & Schulz et al./2000 [41] & 18/yes & $10 /$ yes and $10 /$ no & $\uparrow$ & $\downarrow$ \\
\hline PMN oxidative burst & $\begin{array}{c}\text { Dyugovskaya et al./2002 } \\
\text { [42] }\end{array}$ & 18/yes & $31 /$ yes & $\uparrow$ & $\downarrow$ \\
\hline $\begin{array}{l}\text { PMN p22phox } \\
\text { mRNA }\end{array}$ & Liu et al./2009 [43] & 107/yes & 69/yes & $\uparrow$ & n.a. \\
\hline Serum NOX2 & Del Ben et al./2012 [44] & $91 /$ yes & $47 /$ yes & $\leftrightarrow$ & $\downarrow$ \\
\hline Nitrite and nitrate & Schulz et al./2000 [52] & $21 /$ yes & 18 /yes and $13 /$ no & $\downarrow$ & $\uparrow$ \\
\hline Nitrite and nitrate & Ip et al./2000 [53] & $30 /$ yes & $40 /$ no & $\downarrow$ & $\uparrow$ \\
\hline Nitrite and nitrate & $\begin{array}{l}\text { Alonso-Fernández et } \\
\text { al./2009 [54] }\end{array}$ & $31 /$ no & $15 /$ no & $\downarrow$ & $\uparrow$ \\
\hline ADMA & Barceló et al./2009 [56] & $23 /$ yes and $18 /$ no & $13 /$ no & $\uparrow$ & n.a. \\
\hline Arginase & Yüksel et al./2014 [57] & $41 /$ yes and $10 /$ no & $15 /$ no & $\uparrow$ & n.a. \\
\hline TBARS & Barceló et al./2000 [58] & $14 /$ yes & $13 /$ no & $\uparrow$ & $\downarrow$ \\
\hline TBARS & Lavie et al./2004 [59] & $59 /$ yes and $55 /$ no & $30 /$ yes & $\uparrow$ & $\downarrow$ \\
\hline Exhaled 8-isoprostane & $\begin{array}{c}\text { Carpagnano et al./2003 } \\
{[60]}\end{array}$ & $18 /$ no & $12 /$ no & $\uparrow$ & $\downarrow$ \\
\hline Urinary 8-OHdG & Yamauchi et al./2005 [64] & $128 /$ yes & n.a. & $\uparrow^{*}$ & n.a. \\
\hline Vitamins $\mathrm{A}$ and $\mathrm{E}$ & Barceló et al./2006 [68] & $47 /$ yes & $37 /$ no & $\downarrow$ & $\leftrightarrow$ \\
\hline SOD & Wysocka et al./2008 [70] & $41 /$ no & $39 /$ no & $\downarrow$ & n.a. \\
\hline Paraoxonase-1 & Lavie et al./2004 [59] & $59 /$ yes and $55 /$ no & $30 /$ yes & $\downarrow$ & n.a. \\
\hline
\end{tabular}

$\uparrow$ : increase, $\downarrow$ : decrease, and $\leftrightarrow$ : no change.

* Patients with severe versus mild-to-moderate OSA.

ADMA: asymmetric dimethylarginine.

n.a. = not available.

NOX: NADPH oxidase.

8-OHdG: 8-hydroxy-2' -deoxyguanosine.

PMN: polymorphonuclear neutrophils.

SOD: superoxide dismutase.

TBARS: thiobarbituric acid reactive substances.

infarction or stroke. This could, for instance, be accomplished by enrolling those patients who are noncompliant with CPAP therapy.

The same patient population may also help to establish the role of antioxidants in the treatment of OSA-associated $\mathrm{CV}$ diseases. In this context, it is an open question which antioxidants should be given and at what dose and route of administration. Regardless of these considerations, such studies would need larger patient numbers and long-term follow-up periods to delineate a therapeutic effect of antioxidants in OSA.

In addition to these more clinically oriented questions, research efforts should be undertaken to elucidate the basic mechanisms of oxidative stress in OSA. One possibility to accomplish this task is to go back from bench to bedside, that is, to see if the results of animal studies can be extrapolated to humans with the disease. In this context, numerous studies in mice and rats have shown that an upregulation of NOX underlies many of the CV sequelae of IH [7, 82]. Apart from NOX and its different isoforms, one could also look at other ROS-generating enzymes within the human body as, for example, xanthine oxidase, uncoupled eNOS, and mitochondrial respiratory chain enzymes. Finally, one should try to decipher the interaction of oxidative stress with other putative pathophysiological pathways of CV disease in OSA. In this context, animal studies suggest that ROS can mediate or aggravate sympathetic activation in response to IH [83]. Up to now, it is not known if this is also the case in patients with OSA.

\section{Conclusions}

In summary, there is accumulating evidence from human studies that untreated OSA causes oxidative stress and that effective CPAP therapy can reverse these abnormalities (Figure 2). Given the well-known role of ROS in the pathogenesis of $\mathrm{CV}$ disease, the oxidative stress induced by OSA may account for its associated CV morbidity and mortality; however, more studies are clearly needed in this area of research. In particular, the cardioprotective role of antioxidants in those patients not tolerating CPAP 


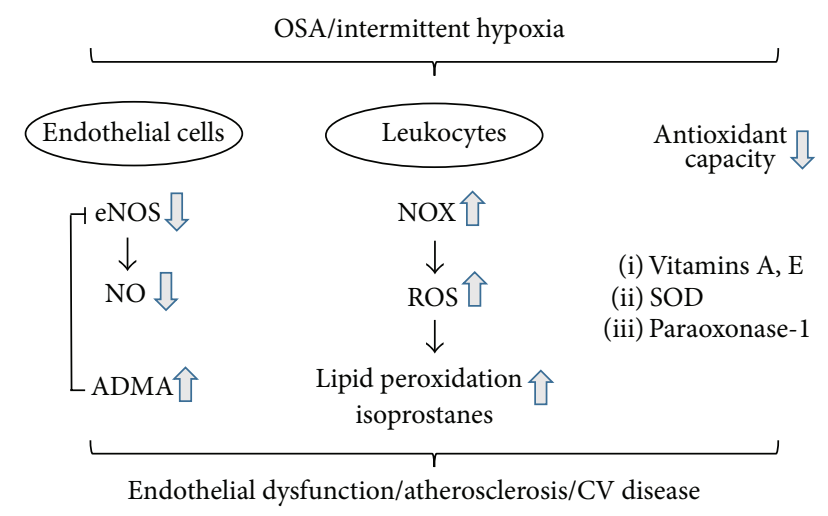

Figure 2: Oxidative stress as an intermediary pathway of OSAassociated CV disease. The intermittent hypoxia characteristic of OSA leads to increased oxidative burst of leukocytes via activation of NOX. Excessively produced ROS enhance lipid peroxidation and isoprostane formation. NO bioavailability is reduced by diminished expression of eNOS and its inhibition by ADMA. Finally, antioxidant capacity is impaired in affected patients. ADMA: asymmetric dimethylarginine; eNOS: endothelial nitric oxide synthase; NO: nitric oxide; NOX: NADPH oxidase; ROS: reactive oxygen species; SOD: superoxide dismutase.

therapy and the basic mechanisms leading to oxidative stress in patients with OSA should be explored in more detail.

\section{Abbreviations}

ADMA: Asymmetric dimethylarginine

BP: $\quad$ Blood pressure

CPAP: Continuous positive airway pressure

CV: Cardiovascular

eNOS: Endothelial nitric oxide synthase

FMD: Flow-mediated vasodilation

IH: Intermittent hypoxia

IMT: Intima-media thickness

NO: $\quad$ Nitric oxide

NOS: Nitric oxide synthase

NOX: NADPH oxidase

OSA: Obstructive sleep apnea

ROS: Reactive oxygen species

SDB: Sleep-disordered breathing

SOD: $\quad$ Superoxide dismutase.

\section{Conflict of Interests}

The authors declare that they have no conflict of interests.

\section{References}

[1] M. Younes, "Role of respiratory control mechanisms in the pathogenesis of obstructive sleep disorders," Journal of Applied Physiology, vol. 105, no. 5, pp. 1389-1405, 2008.

[2] T. Young, P. E. Peppard, and D. J. Gottlieb, "Epidemiology of obstructive sleep apnea: a population health perspective,"
American Journal of Respiratory and Critical Care Medicine, vol. 165, no. 9, pp. 1217-1239, 2002.

[3] T. Young, M. Palta, J. Dempsey, J. Skatrud, S. Weber, and S. Badr, "The occurrence of sleep-disordered breathing among middleaged adults," The New England Journal of Medicine, vol. 328, no. 17, pp. 1230-1235, 1993.

[4] C. E. Woods, K. Usher, and G. P. Maguire, "Obstructive sleep apnoea in adult indigenous populations in high-income countries: an integrative review," Sleep and Breathing, vol. 19, no. 1, pp. 45-53, 2015.

[5] J. M. Marin, S. J. Carrizo, E. Vicente, and A. G. N. Agusti, "Longterm cardiovascular outcomes in men with obstructive sleep apnoea-hypopnoea with or without treatment with continuous positive airway pressure: an observational study," The Lancet, vol. 365, no. 9464, pp. 1046-1053, 2005.

[6] C. E. Sullivan, F. G. Issa, M. Berthon-Jones, and L. Eves, "Reversal of obstructive sleep apnoea by continuous positive airway pressure applied through the nares," The Lancet, vol. 1, no. 8225, pp. 862-865, 1981.

[7] R. Dumitrascu, J. Heitmann, W. Seeger, N. Weissmann, and R. Schulz, "Obstructive sleep apnea, oxidative stress and cardiovascular disease: lessons from animal studies," Oxidative Medicine and Cellular Longevity, vol. 2013, Article ID 234631, 7 pages, 2013.

[8] O. Marrone and M. R. Bonsignore, "Pulmonary haemodynamics in obstructive sleep apnoea," Sleep Medicine Reviews, vol. 6, no. 3, pp. 175-193, 2002.

[9] C. Guilleminault, S. Connolly, and R. Winkle, "Cyclical variation of the heart rate in sleep apnoea syndrome. Mechanisms, and usefulness of $24 \mathrm{~h}$ electrocardiography as a screening technique," The Lancet, vol. 1, no. 8369, pp. 126-131, 1984.

[10] V. A. Rossi, J. R. Stradling, and M. Kohler, "Effects of obstructive sleep apnoea on heart rhythm," European Respiratory Journal, vol. 41, no. 6, pp. 1439-1451, 2013.

[11] A. S. Gami, E. J. Olson, W. K. Shen et al., "Obstructive sleep apnea and the risk of sudden cardiac death: a longitudinal study of 10,701 adults," Journal of the American College of Cardiology, vol. 62, no. 7, pp. 610-616, 2013.

[12] J. Wolf, D. Hering, and K. Narkiewicz, "Non-dipping pattern of hypertension and obstructive sleep apnea syndrome," Hypertension Research, vol. 33, no. 9, pp. 867-871, 2010.

[13] A. G. Logan, S. M. Perlikowski, A. Mente et al., "High prevalence of unrecognized sleep apnoea in drug-resistant hypertension," Journal of Hypertension, vol. 19, no. 12, pp. 2271-2277, 2001.

[14] J.-P. Baguet, P. Lévy, G. Barone-Rochette et al., "Masked hypertension in obstructive sleep apnea syndrome," Journal of Hypertension, vol. 26, no. 5, pp. 885-892, 2008.

[15] R. H. Mason, G. Ruegg, J. Perkins et al., "Obstructive sleep apnea in patients with abdominal aortic aneurysms: highly prevalent and associated with aneurysm expansion," American Journal of Respiratory and Critical Care Medicine, vol. 183, no. 5, pp. 668674, 2011.

[16] D. Sajkov, T. Wang, N. A. Saunders, A. J. Bune, A. M. Neill, and R. D. McEvoy, "Daytime pulmonary hemodynamics in patients with obstructive sleep apnea without lung disease," American Journal of Respiratory and Critical Care Medicine, vol. 159, no. 5 I, pp. 1518-1526, 1999.

[17] M. Arzt, R. Luigart, C. Schum et al., "Sleep-disordered breathing in deep vein thrombosis and acute pulmonary embolism," European Respiratory Journal, vol. 40, no. 4, pp. 919-924, 2012. 
[18] J. T. Carlson, C. Rångemark, and J. A. Hedner, "Attenuated endothelium-dependent vascular relaxation in patients with sleep apnoea," Journal of Hypertension, vol. 14, no. 5, pp. 577584, 1996.

[19] R. Schulz, W. Seeger, C. Fegbeutel et al., "Changes in extracranial arteries in obstructive sleep apnoea," European Respiratory Journal, vol. 25, no. 1, pp. 69-74, 2005.

[20] F. Campos-Rodriguez, M. A. Martinez-Garcia, I. de la CruzMoron, C. Almeida-Gonzalez, P. Catalan-Serra, and J. M. Montserrat, "Cardiovascular mortality in women with obstructive sleep apnea with or without continuous positive airway pressure treatment: a cohort study," Annals of Internal Medicine, vol. 156, no. 2, pp. 115-122, 2012.

[21] M.-A. Martínez-García, F. Campos-Rodríguez, P. Catalán-Serra et al., "Cardiovascular mortality in obstructive sleep apnea in the elderly: role of long-term continuous positive airway pressure treatment: a prospective observational study," American Journal of Respiratory and Critical Care Medicine, vol. 186, no. 9, pp. 909-916, 2012.

[22] F. P. Cappuccio, D. Cooper, L. D'Elia, P. Strazzullo, and M. A. Miller, "Sleep duration predicts cardiovascular outcomes: a systematic review and meta-analysis of prospective studies," European Heart Journal, vol. 32, no. 12, pp. 1484-1492, 2011.

[23] F. Sofi, F. Cesari, A. Casini, C. Macchi, R. Abbate, and G. F. Gensini, "Insomnia and risk of cardiovascular disease: a metaanalysis," European Journal of Preventive Cardiology, vol. 21, no. 1, pp. 57-64, 2014.

[24] M. A. Gupta and K. Knapp, "Cardiovascular and psychiatric morbidity in Obstructive Sleep Apnea (OSA) with Insomnia (sleep apnea plus) versus obstructive sleep apnea without insomnia: a case-control study from a nationally representative US sample," PLoS ONE, vol. 9, no. 3, Article ID e90021, 2014.

[25] V. K. Somers, M. E. Dyken, M. P. Clary, and F. M. Abboud, "Sympathetic neural mechanisms in obstructive sleep apnea," The Journal of Clinical Investigation, vol. 96, no. 4, pp. 1897-1904, 1995.

[26] A. K. Htoo, H. Greenberg, S. Tongia et al., "Activation of nuclear factor kappaB in obstructive sleep apnea: a pathway leading to systemic inflammation," Sleep and Breathing, vol. 10, no. 1, pp. 43-50, 2006.

[27] S. A. Gharib, A. L. Hayes, M. J. Rosen, and S. R. Patel, "A pathway-based analysis on the effects of obstructive sleep apnea in modulating visceral fat transcriptome," Sleep, vol. 36, no. 1, pp. 23-30, 2013.

[28] B. G. Phillips, K. Narkiewicz, C. A. Pesek, W. G. Haynes, M. E. Dyken, and V. K. Somers, "Effects of obstructive sleep apnea on endothelin-1 and blood pressure," Journal of Hypertension, vol. 17, no. 1, pp. 61-66, 1999.

[29] R. Schulz, C. Hummel, S. Heinemann, W. Seeger, and F. Grimminger, "Serum levels of vascular endothelial growth factor are elevated in patients with obstructive sleep apnea and severe nighttime hypoxia," American Journal of Respiratory and Critical Care Medicine, vol. 165, no. 1, pp. 67-70, 2002.

[30] G. Bokinsky, M. Miller, K. Ault, P. Husband, and J. Mitchell, "Spontaneous platelet activation and aggregation during obstructive sleep apnea and its response to therapy with nasal continuous positive airway pressure. A preliminary investigation," Chest, vol. 108, no. 3, pp. 625-630, 1995.

[31] M. de la Peña, A. Barceló, F. Barbe et al., "Endothelial function and circulating endothelial progenitor cells in patients with sleep apnea syndrome," Respiration, vol. 76, no. 1, pp. 28-32, 2008.
[32] M. S. M. Ip, B. Lam, M. M. T. Ng, W. K. Lam, K. W. T. Tsang, and K. S. L. Lam, "Obstructive sleep apnea is independently associated with insulin resistance," American Journal of Respiratory and Critical Care Medicine, vol. 165, no. 5, pp. 670-676, 2002.

[33] A. M. Adedayo, O. Olafiranye, D. Smith et al., "Obstructive sleep apnea and dyslipidemia: evidence and underlying mechanism," Sleep and Breathing, vol. 18, no. 1, pp. 13-18, 2014.

[34] H. Sies, "Oxidative stress: a concept in redox biology and medicine," Redox Biology, vol. 4, pp. 180-183, 2015.

[35] B. Halliwell, "Free radicals, antioxidants, and human disease: curiosity, cause, or consequence?” The Lancet, vol. 344, no. 8924, pp. 721-724, 1994.

[36] B. Halliwell, "The role of oxygen radicals in human disease, with particular reference to the vascular system," Haemostasis, vol. 23, no. 1, pp. 118-126, 1993.

[37] S. Cofta, E. Wysocka, T. Piorunek, M. Rzymkowska, H. BaturaGabryel, and L. Torlinski, "Oxidative stress markers in the blood of persons with different stages of obstructive sleep apnea syndrome," Journal of Physiology and Pharmacology, vol. 59, no. 6, pp. 183-190, 2008.

[38] K. Katsoulis, T. Kontakiotis, D. Spanogiannis et al., "Total antioxidant status in patients with obstructive sleep apnea without comorbidities: the role of the severity of the disease," Sleep and Breathing, vol. 15, no. 4, pp. 861-866, 2011.

[39] C. Papandreou, "Levels of TBARS are inversely associated with lowest oxygen saturation in obese patients with OSAS," Sleep and Breathing, vol. 17, no. 4, pp. 1319-1322, 2013.

[40] A. Svatikova, R. Wolk, L. O. Lerman et al., "Oxidative stress in obstructive sleep apnoea," European Heart Journal, vol. 26, no. 22, pp. 2435-2439, 2005.

[41] R. Schulz, S. Mahmoudi, K. Hattar et al., "Enhanced release of superoxide from polymorphonuclear neutrophils in obstructive sleep apnea: impact of continuous positive airway pressure therapy," American Journal of Respiratory and Critical Care Medicine, vol. 162, no. 2, part 1, pp. 566-570, 2000.

[42] L. Dyugovskaya, P. Lavie, and L. Lavie, "Increased adhesion molecules expression and production of reactive oxygen species in leukocytes of sleep apnea patients," American Journal of Respiratory and Critical Care Medicine, vol. 165, no. 7, pp. 934939, 2002.

[43] H.-G. Liu, K. Liu, Y.-N. Zhou, and Y.-J. Xu, "Relationship between reduced nicotinamide adenine dinucleotide phosphate oxidase subunit p22phox gene polymorphism and obstructive sleep apnea-hypopnea syndrome in the Chinese Han population," Chinese Medical Journal, vol. 122, no. 12, pp. 1369-1374, 2009.

[44] M. Del Ben, M. Fabiani, L. Loffredo et al., "Oxidative stress mediated arterial dysfunction in patients with obstructive sleep apnoea and the effect of continuous positive airway pressure treatment," BMC Pulmonary Medicine, vol. 12, article 36, 2012.

[45] P. J. Andrew and B. Mayer, "Enzymatic function of nitric oxide synthases," Cardiovascular Research, vol. 43, no. 3, pp. 521-531, 1999.

[46] J. L. Boucher, C. Moali, and J. P. Tenu, "Nitric oxide biosynthesis, nitric oxide synthase inhibitors and arginase competition for Larginine utilization," Cellular and Molecular Life Sciences, vol. 55, no. 8-9, pp. 1015-1028, 1999.

[47] S. Imai, "Cyclic GMP as a second messenger in the cardiovascular system," Japanese Heart Journal, vol. 36, no. 2, pp. 127-177, 1995. 
[48] R. A. Cohen, "The role of nitric oxide and other endotheliumderived vasoactive substances in vascular disease," Progress in Cardiovascular Diseases, vol. 38, no. 2, pp. 105-128, 1995.

[49] W. H. Koppenol, J. J. Moreno, W. A. Pryor, H. Ischiropoulos, and J. S. Beckman, "Peroxynitrite, a cloaked oxidant formed by nitric oxide and superoxide," Chemical Research in Toxicology, vol. 5, no. 6, pp. 834-842, 1992.

[50] Z. S. Katusic, "Vascular endothelial dysfunction: does tetrahydrobiopterin play a role?" American Journal of PhysiologyHeart and Circulatory Physiology, vol. 281, no. 3, pp. H981H986, 2001.

[51] F. Arrigoni, B. Ahmetaj, and J. Leiper, "The biology and therapeutic potential of the DDAH/ADMA pathway," Current Pharmaceutical Design, vol. 16, no. 37, pp. 4089-4102, 2010.

[52] R. Schulz, D. Schmidt, A. Blum et al., "Decreased plasma levels of nitric oxide derivatives in obstructive sleep apnoea: response to CPAP therapy," Thorax, vol. 55, no. 12, pp. 1046-1051, 2000.

[53] M. S. M. Ip, B. Lam, L.-Y. Chan et al., "Circulating nitric oxide is suppressed in obstructive sleep apnea and is reversed by nasal continuous positive airway pressure," American Journal of Respiratory and Critical Care Medicine, vol. 162, no. 6, pp. 21662171, 2000.

[54] A. Alonso-Fernández, F. García-Río, M. A. Arias et al., "Effects of CPAP on oxidative stress and nitrate efficiency in sleep apnoea: a randomised trial," Thorax, vol. 64, no. 7, pp. 581-586, 2009.

[55] Y. Ozkan, H. Firat, B. Şimşek, M. Torun, and S. Yardim-Akaydin, "Circulating nitric oxide (NO), asymmetric dimethylarginine (ADMA), homocysteine, and oxidative status in obstructive sleep apnea-hypopnea syndrome (OSAHS)," Sleep and Breathing, vol. 12, no. 2, pp. 149-154, 2008.

[56] A. Barceló, M. de la Peña, O. Ayllón et al., "Increased plasma levels of asymmetric dimethylarginine and soluble CD40 ligand in patients with sleep apnea," Respiration, vol. 77, no. 1, pp. 8590, 2009.

[57] M. Yüksel, H. K. Okur, Z. Pelin, A. V. Öğünç, and L. Öztürk, "Arginase activity and nitric oxide levels in patients with obstructive sleep apnea syndrome," Clinics, vol. 69, no. 4, pp. 247-252, 2014.

[58] A. Barceló, C. Miralles, F. Barbé, M. Vila, S. Pons, and A. G. N. Agustí, "Abnormal lipid peroxidation in patients with sleep apnoea," European Respiratory Journal, vol. 16, no. 4, pp. 644$647,2000$.

[59] L. Lavie, A. Vishnevsky, and P. Lavie, "Evidence for lipid peroxidation in obstructive sleep apnea," Sleep, vol. 27, no. 1, pp. 123-128, 2004.

[60] G. E. Carpagnano, S. A. Kharitonov, O. Resta, M. P. FoschinoBarbaro, E. Gramiccioni, and P. J. Barnes, "8-Isoprostane, a marker of oxidative stress, is increased in exhaled breath condensate of patients with obstructive sleep apnea after night and is reduced by continuous positive airway pressure therapy," Chest, vol. 124, no. 4, pp. 1386-1392, 2003.

[61] G. L. Milne, Q. Dai, and L. J. Roberts II, “The isoprostanes-25 years later," Biochimica et Biophysica Acta, vol. 1851, no. 4, pp. 433-445, 2015.

[62] S. Ozben, N. Huseyinoglu, F. Hanikoglu et al., "Advanced oxidation protein products and ischaemia-modified albumin in obstructive sleep apnea," European Journal of Clinical Investigation, vol. 44, no. 11, pp. 1045-1052, 2014.

[63] E. Hopps, B. Canino, V. Calandrino, M. Montana, R. Lo Presti, and G. Caimi, "Lipid peroxidation and protein oxidation are related to the severity of OSAS," European Review for Medical and Pharmacological Sciences, vol. 18, no. 24, pp. 3773-3778, 2014.

[64] M. Yamauchi, H. Nakano, J. Maekawa et al., "Oxidative stress in obstructive sleep apnea," Chest, vol. 127, no. 5, pp. 1674-1679, 2005.

[65] J. Xie, J. Jiang, K. Shi et al., "DNA damage in peripheral blood lymphocytes from patients with OSAHS," Sleep and Breathing, vol. 18, no. 4, pp. 775-780, 2014.

[66] Y. S. Kim, J. W. Kwak, K. E. Lee et al., "Can mitochondrial dysfunction be a predictive factor for oxidative stress in patients with obstructive sleep apnea?" Antioxidants \& Redox Signaling, vol. 21, no. 9, pp. 1285-1288, 2014.

[67] K. Christou, A. N. Moulas, C. Pastaka, and K. I. Gourgoulianis, "Antioxidant capacity in obstructive sleep apnea patients," Sleep Medicine, vol. 4, no. 3, pp. 225-228, 2003.

[68] A. Barceló, F. Barbé, M. de la Peña et al., "Antioxidant status in patients with sleep apnoea and impact of continuous positive airway pressure treatment," European Respiratory Journal, vol. 27, no. 4, pp. 756-760, 2006.

[69] P. Faure, R. Tamisier, J.-P. Baguet et al., "Impairment of serum albumin antioxidant properties in obstructive sleep apnoea syndrome," European Respiratory Journal, vol. 31, no. 5, pp. 1046-1053, 2008.

[70] E. Wysocka, S. Cofta, M. Cymerys, J. Gozdzik, L. Torlinski, and H. Batura-Gabryel, "The impact of the sleep apnea syndrome on oxidant-antioxidant balance in the blood of overweight and obese patients," Journal of Physiology and Pharmacology, vol. 59, supplement 6, pp. 761-769, 2008.

[71] M. S. Hoffmann, P. Singh, R. Wolk, A. Romero-Corral, S. Raghavakaimal, and V. K. Somers, "Microarray studies of genomic oxidative stress and cell cycle responses in obstructive sleep apnea," Antioxidants and Redox Signaling, vol. 9, no. 6, pp. 661-669, 2007.

[72] S. Jelic, M. Padeletti, S. M. Kawut et al., "Inflammation, oxidative stress, and repair capacity of the vascular endothelium in obstructive sleep apnea," Circulation, vol. 117, no. 17, pp. 22702278, 2008.

[73] B. T. Patt, D. Jarjoura, D. N. Haddad et al., "Endothelial dysfunction in the microcirculation of patients with obstructive sleep apnea," The American Journal of Respiratory and Critical Care Medicine, vol. 182, no. 12, pp. 1540-1545, 2010.

[74] S. Varadharaj, K. Porter, A. Pleister et al., "Endothelial nitric oxide synthase uncoupling: a novel pathway in OSA induced vascular endothelial dysfunction," Respiratory Physiology \& Neurobiology, vol. 207, pp. 40-47, 2015.

[75] M. S. M. Ip, H.-F. Tse, B. Lam, K. W. T. Tsang, and W.-K. Lam, "Endothelial function in obstructive sleep apnea and response to treatment," American Journal of Respiratory and Critical Care Medicine, vol. 169, no. 3, pp. 348-353, 2004.

[76] Y. Ohike, K. Kozaki, K. Iijima et al., "Amelioration of vascular endothelial dysfunction in obstructive sleep apnea syndrome by nasal continuous positive airway pressure: possible involvement of nitric oxide and asymmetric NG, NG-dimethylarginine," Circulation Journal, vol. 69, no. 2, pp. 221-226, 2005.

[77] L. Loffredo, A. M. Zicari, F. Occasi et al., "Endothelial dysfunction and oxidative stress in children with sleep disordered breathing: role of NADPH oxidase," Atherosclerosis, vol. 240, no. 1, pp. 222-227, 2015.

[78] D. Monneret, J.-L. Pepin, D. Godin-Ribuot et al., "Association of urinary 15-F2t-isoprostane level with oxygen desaturation 
and carotid intima-media thickness in nonobese sleep apnea patients," Free Radical Biology and Medicine, vol. 48, no. 4, pp. 619-625, 2010.

[79] M. Grebe, H. J. Eisele, N. Weissmann et al., "Antioxidant vitamin C improves endothelial function in obstructive sleep apnea," American Journal of Respiratory and Critical Care Medicine, vol. 173, no. 8, pp. 897-901, 2006.

[80] N. J. Büchner, I. Quack, M. Woznowski, C. Stähle, U. Wenzel, and L. C. Rump, "Microvascular endothelial dysfunction in obstructive sleep apnea is caused by oxidative stress and improved by continuous positive airway pressure therapy," Respiration, vol. 82, no. 5, pp. 409-417, 2011.

[81] A. A. El Solh, R. Saliba, T. Bosinski, B. J. B. Grant, E. Berbary, and N. Miller, "Allopurinol improves endothelial function in sleep apnoea: a randomised controlled study," European Respiratory Journal, vol. 27, no. 5, pp. 997-1002, 2006.

[82] R. Schulz, G. Murzabekova, B. Egemnazarov et al., "Arterial hypertension in a murine model of sleep apnea: role of NADPH oxidase 2," Journal of Hypertension, vol. 32, no. 2, pp. 300-305, 2014.

[83] N. R. Prabhakar, G. K. Kumar, and Y.-J. Peng, "Sympathoadrenal activation by chronic intermittent hypoxia," Journal of Applied Physiology, vol. 113, no. 8, pp. 1304-1310, 2012. 


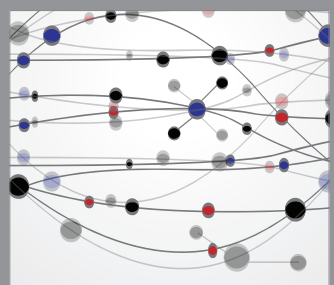

The Scientific World Journal
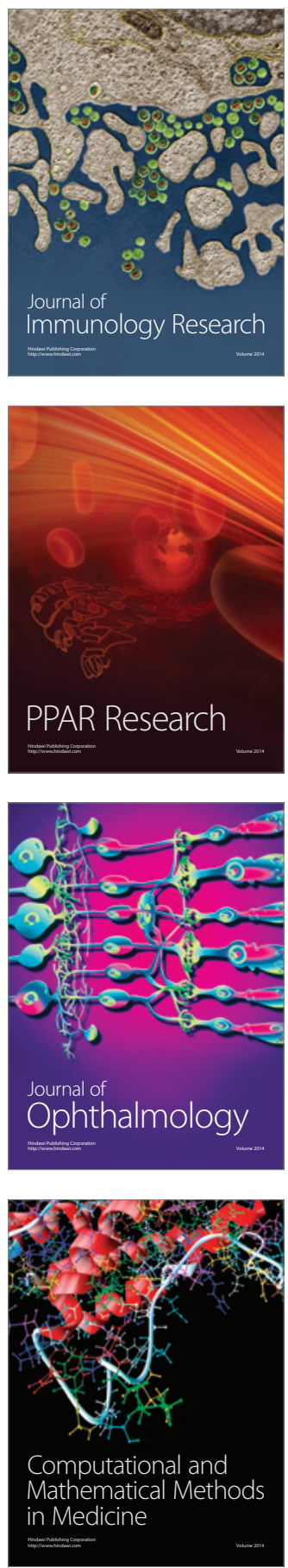

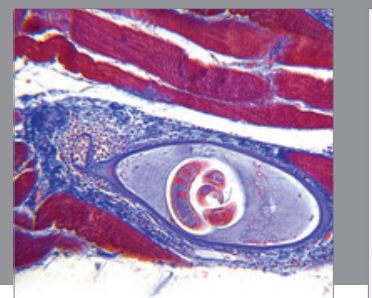

Gastroenterology

Research and Practice
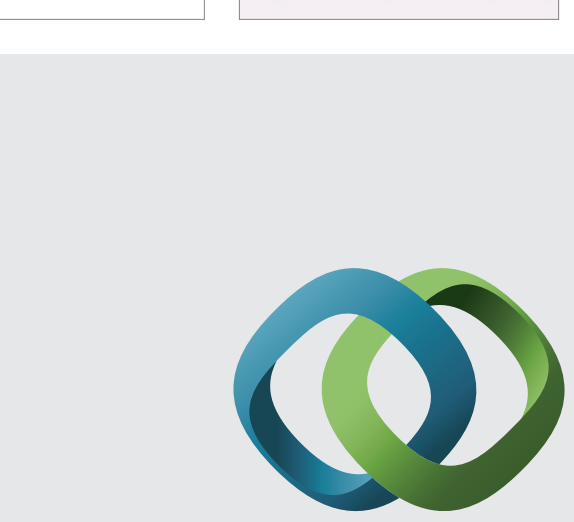

\section{Hindawi}

Submit your manuscripts at

http://www.hindawi.com
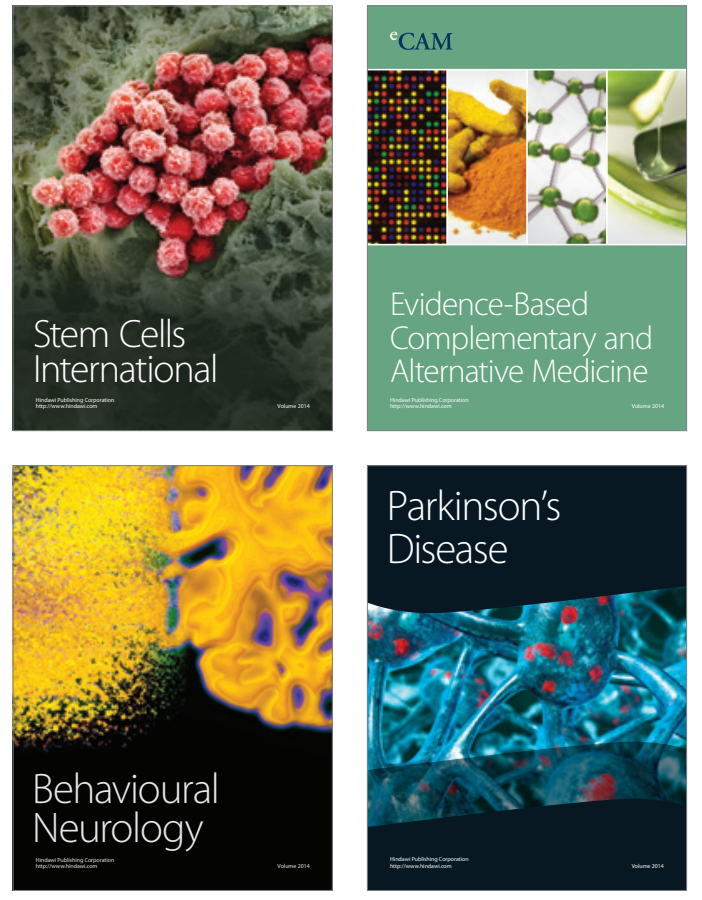
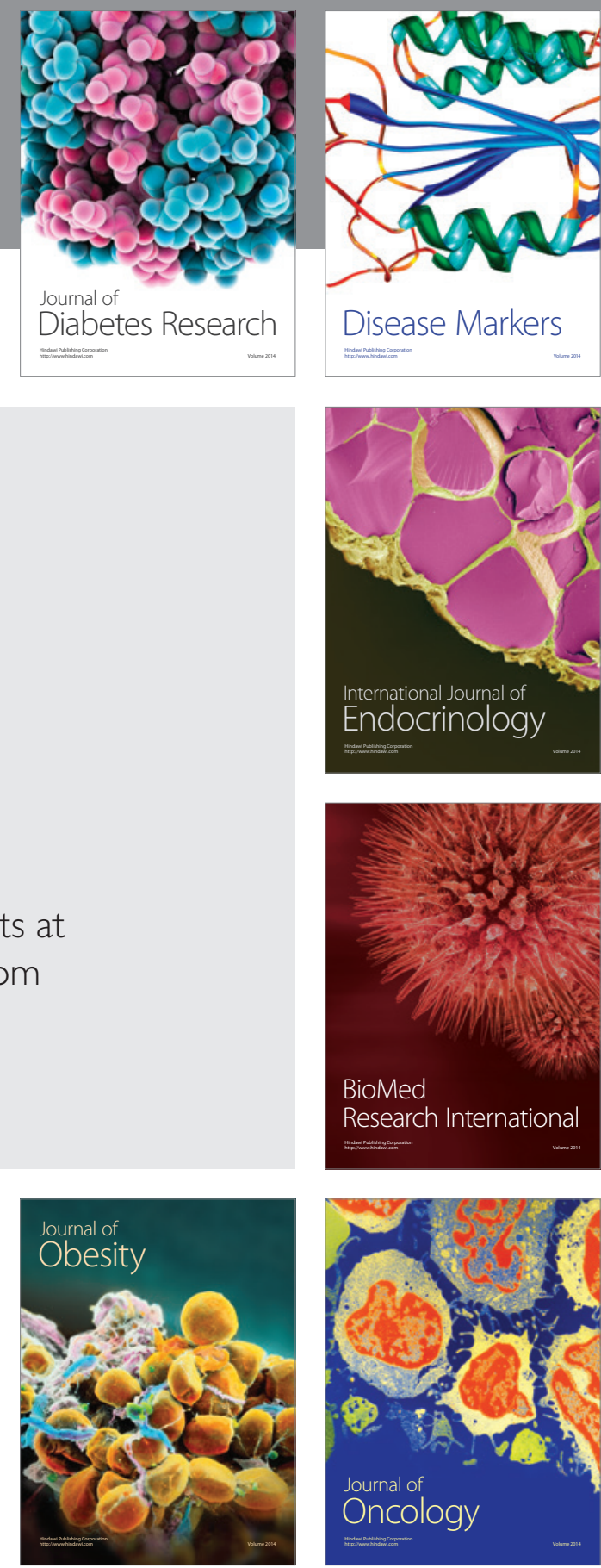

Disease Markers
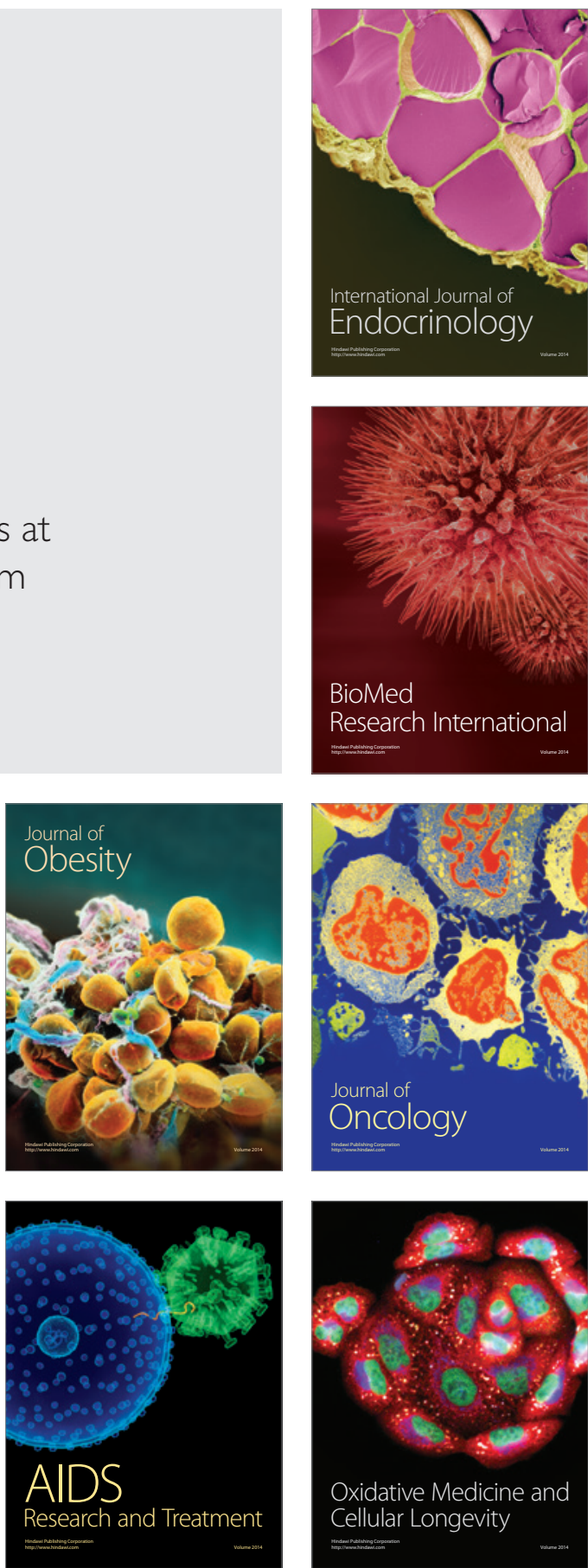